\title{
Palliative care and good death in acute diseases: a scoping
}

\section{review protocol [version 1; peer review: 1 not approved]}

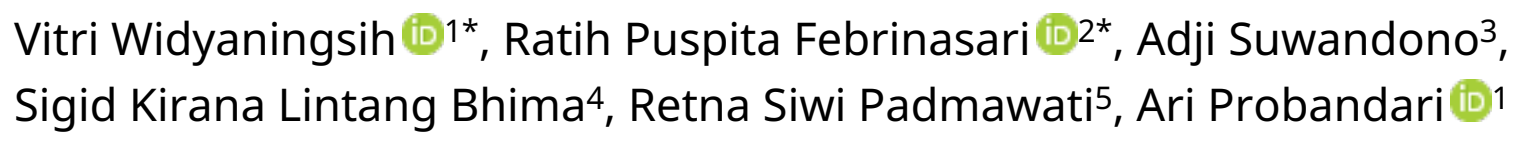

${ }^{1}$ Public Health, Universitas Sebelas Maret, Surakarta, Central Java, 57126, Indonesia

2Pharmacology, Universitas Sebelas Maret, Surakarta, Central Java, 57126, Indonesia

${ }^{3}$ Doctoral Program of Public Health, Universitas Sebelas Maret, Surakarta, Central Java, 57126, Indonesia

${ }^{4}$ Forensic and Legal Medicine, Diponegoro University, Semarang, Central Java, 50275, Indonesia

${ }^{5}$ Centre for Bioethics and Medical Humanities, Faculty of Medicine, Public Health, and Nursing, Universitas Gadjah Mada, Yogyakarta, 55281, Indonesia

* Equal contributors

V1 First published: 12 Nov 2021, 10:1147

https://doi.org/10.12688/f1000research.74403.1

Latest published: 13 Apr 2022, 10:1147

https://doi.org/10.12688/f1000research.74403.2

\section{Abstract}

Increasing cases of emerging and re-emerging infectious diseases, requires healthcare systems to provide essential palliative care for critically ill patients and their families. With the rapid onset and often accelerated deterioration in patients with acute conditions, palliative and supportive care for these patients have different characteristics compared to those for chronic diseases. Furthermore, providing endof life services for critically ill patients with acute diseases and their families to ensure good death for the patients, will also have its own challenges. This scoping review aims to explore the concept of palliative care and good death for acute diseases.

This scoping review will be conducted using the Arksey and O'Malley's framework for scoping reviews: identifying the research question, identifying relevant studies, study selection, charting the data, collating, summarizing, reporting results, and conducting consultation. All original research with a focus on palliative care and good death due to acute diseases will be included. This review will include all original research designs published between the period of 2000-2021 that describe a measure of palliative care management for and good death due to acute diseases. Quantitative, qualitative and mixed-method studies will be included in order to consider different aspects of healthcare services. This review will also include guidelines and gray literature on palliative care and good deaths. The search will be conducted through PubMed, Scopus Database, and ScienceDirect using the key terms related to acute disease palliative care and the concept of good death due to acute diseases. Two authors will screen the titles and abstracts of the studies. Two authors will review the full text of selected studies independently and extract the data. All

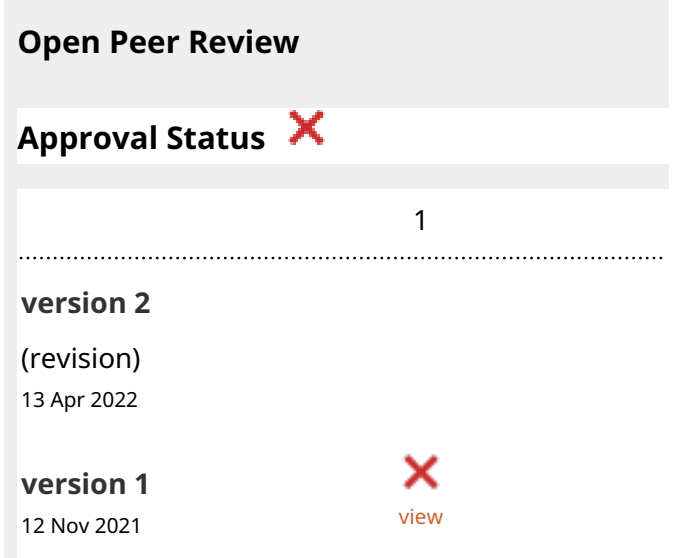

1. Hanan Khalil ID, La Trobe University,
Melbourne, Australia

Any reports and responses or comments on the article can be found at the end of the article. 
selected studies will be synthesized qualitatively, and the results will be consulted with experts through discussion and depict the current concept of palliative care and good death in acute diseases.

\section{Keywords}

good death, palliative care, acute disease, infectious disease, health systems

\section{Corresponding author: Ari Probandari (ari.probandari@staff.uns.ac.id)}

Author roles: Widyaningsih V: Conceptualization, Data Curation, Formal Analysis, Investigation, Methodology, Resources, Software, Supervision, Validation, Visualization, Writing - Original Draft Preparation; Febrinasari RP: Conceptualization, Data Curation, Formal Analysis, Investigation, Methodology, Project Administration, Resources, Software, Supervision, Validation, Visualization, Writing Review \& Editing; Suwandono A: Data Curation, Formal Analysis, Investigation, Resources, Visualization, Writing - Review \& Editing; Bhima SKL: Conceptualization, Data Curation, Formal Analysis, Funding Acquisition, Methodology, Supervision, Writing - Review \& Editing; Padmawati RS: Conceptualization, Data Curation, Formal Analysis, Funding Acquisition, Methodology, Resources, Supervision, Validation, Writing - Review \& Editing; Probandari A: Conceptualization, Data Curation, Formal Analysis, Funding Acquisition, Investigation, Methodology, Resources, Supervision, Validation, Writing - Review \& Editing

Competing interests: No competing interests were disclosed.

Grant information: This work was supported by the Ministry of Research and Technology/National Agency for Research and Innovation (RISTEK-BRIN).

The funders had no role in study design, data collection and analysis, decision to publish, or preparation of the manuscript.

Copyright: @ 2021 Widyaningsih V et al. This is an open access article distributed under the terms of the Creative Commons Attribution License, which permits unrestricted use, distribution, and reproduction in any medium, provided the original work is properly cited.

How to cite this article: Widyaningsih V, Febrinasari RP, Suwandono A et al. Palliative care and good death in acute diseases: a scoping review protocol [version 1; peer review: 1 not approved] F1000Research 2021, 10:1147

https://doi.org/10.12688/f1000research.74403.1

First published: 12 Nov 2021, 10:1147 https://doi.org/10.12688/f1000research.74403.1 


\section{Introduction}

Palliative care is provided for a person with an active, progressive and advanced disease who is in serious or lifethreatening health conditions. ${ }^{1,2}$ Palliative care aims to optimize the quality of life and addresses physical suffering of the patient. Furthermore, the palliative care also aims to improve psychological, social and spiritual conditions for patients and their family members. ${ }^{1-3}$ Palliative care involves a multidisciplinary team beyond healthcare professionals, including spiritual, psychology, and/or social worker team member. ${ }^{4-6}$ Palliative care is designed as a person-centered and familycentered care, addressing each patient's and family members specific conditions and needs. ${ }^{7,8}$ There are two types of palliative care, namely terminal palliative care which focuses on the treatment of immediate disease symptoms, and early palliative care that provides patients and family members support for coping with the diagnosis and helps balance decision-making between values and preferences of the patient and realistic expectations of the outcome of treatment. ${ }^{9}$ In low to middle income countries, dissimilarity of access to palliative care shows great disparities in global health care. ${ }^{2,10}$ The concept of palliative care is more commonly discussed and studied in chronic diseases, however, there is still lacking evidence on palliative care on acute diseases.

Acute diseases, such as infections, injuries, acute exacerbations of chronic illnesses, are sudden, rapid and severe diseases that last for a brief time period. ${ }^{11,12}$ High mortality rate might be found on acute emergency admission, especially amongst the elderly with multiple co-morbid conditions. ${ }^{13}$ Therefore, palliative care should be an important component in acute disease management, particularly for severe cases.

The concept of palliative care is also closely related to perceived good death. Good death or successful dying is defined as the person's preferences for the dying process. These preferences might include how, where, when, pain-free status of dying process. ${ }^{14,15}$ The concept of good death also include who accompanied the person during the dying process, and the manner of facing death (i.e. awareness and readiness of the dying process, natural or sudden death). ${ }^{14-16}$ Ironically, studies have shown that patients with contagious disease have been isolated from their family members due to hospital policies and many of them have a deep fear of dying alone. ${ }^{15,17}$ Majority of people want to be with their loved ones when their life comes to an end. ${ }^{15,18}$

Up until now, there have been limited summaries on the experience of palliative care and concept of good death in acute diseases. Further, there is a lack of information on the ideal concept of palliative care and good death in acute disease from the perspective of patients, family or caregivers, as well as healthcare providers. This scoping review aims to explore the concept of palliative care and good death for acute diseases from the perspective of patients, families, and providers, hence, depicting the existing palliative care and good death concepts, and providing recommendation on the ideal concept of palliative care and good death in acute diseases.

\section{Protocol}

To conduct this scoping review, the framework by Arksey and O'Malley will be used. ${ }^{19}$ The framework consist of six steps: 1) identification of research questions; 2) identification of relevant studies; 3) selection of relevant studies; 4) data extraction and charting, 5) summary, analyses, and reporting; and 6) consultation with relevant stakeholders. ${ }^{19}$ The search process, database creation and data extraction will be conducted from September to October 2021. The analysis and reporting will be carried out in December 2021 - April 2022.

\section{Step 1: Identification of research questions}

In this scoping review, we aim to assess the concept of palliative care and good death in acute illness. The research questions can be further elaborated as follows:

1. What is the experience of patients, family of patients, and healthcare providers regarding palliative care for acute diseases?

2. What is the experience of patients, family of patients, and healthcare providers regarding good death due to acute illness?"

3. What is the perception of patients, family of patients, and healthcare providers regarding an ideal palliative care for patients with acute diseases?

4. What is the perception of patients, family of patients, and healthcare providers regarding what is considered as good deaths among patients with acute diseases? 
Step 2: Identification of relevant studies

This scoping review includes the relevant studies based on the inclusion and selection criteria shown on Table 1. The format followed the Joanna Briggs Institute (JBI) protocol. ${ }^{20}$

The search strategy using keywords and queries can be found in Table 2 . The literature search will be conducted in three databases (Pubmed, Scopus, and Science Direct) for articles published between January 2000 to October 2021, hence, providing a review of literature for the past 20 years.

The search strategy will use Medical Subject Heading (MesH) terms: "Acute Disease", "Palliative Care", and "Good Death" on the Pubmed database. An initial search with synonyms of those keywords will be used in the Scopus and ScienceDirect database. Next, analysis of the words contained in the title and abstract will be done. Clinical guidelines database and gray literature will be included and listed in Figure 1.

\section{Step 3: Selection of relevant studies}

Two authors will screen the titles and abstracts of studies according to the selection criteria. In the scoping review, we will include all studies that evaluate the acute disease palliative care and good death. We will exclude studies focusing on chronic disease. Two authors will review the full text of selected studies independently. We will resolve disagreements on study selection and data extraction by discussion with one more reviewer if needed. The details of the study selection are depicted in Figure 2.

\section{Step 4: Data extraction and charting}

The two reviewers will independently chart the data, discuss the results and continuously update the data-charting form in an iterative process. Data extraction will be carried out following the form that has been prepared in Table 3 .

\section{Table 1. Inclusion and exclusion criteria.}

\begin{tabular}{|c|c|c|c|}
\hline Population & Concept & Context & Type of sources \\
\hline
\end{tabular}

Table 2. Keywords and queries for search strategy of acute disease palliative care and good death management (screening, diagnosis, treatment).

\begin{tabular}{|c|c|}
\hline Topic & Keywords and queries \\
\hline $\begin{array}{l}\text { Palliative } \\
\text { case }\end{array}$ & $\begin{array}{l}\text { ("palliative care" [MeSH Terms] OR "palliative care" [All Fields] OR "supportive care" [All Fields]) AND } \\
\text { ((("acute" [All Fields] OR "acutely" [All Fields] OR "acutes" [All Fields]) AND "infections" [MeSH Terms]) } \\
\text { OR "acute infection" [All Fields]) } \\
\text { ("palliative care" [MeSH Terms] OR "palliative care" [All Fields] OR "supportive care" [All Fields]) AND } \\
\text { ("communicable diseases" [MeSH Terms] OR "infectious disease" [All Fields]) }\end{array}$ \\
\hline Good death & 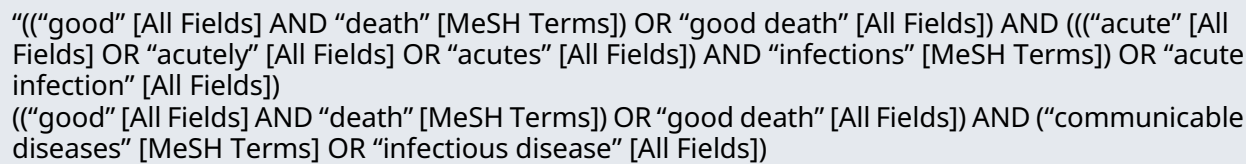 \\
\hline
\end{tabular}




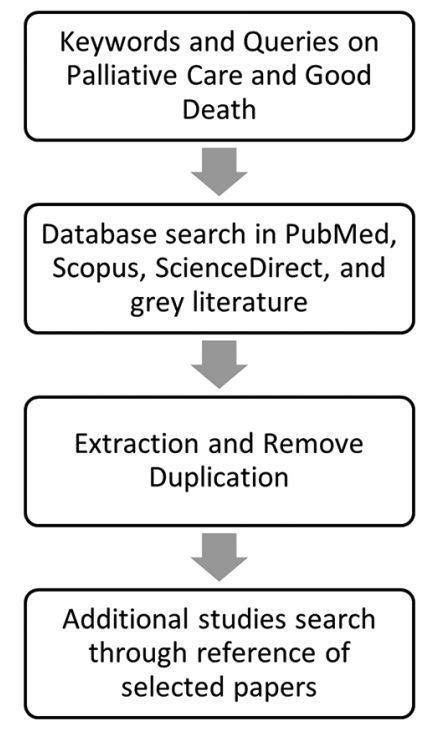

Figure 1. Steps of the searching strategy.

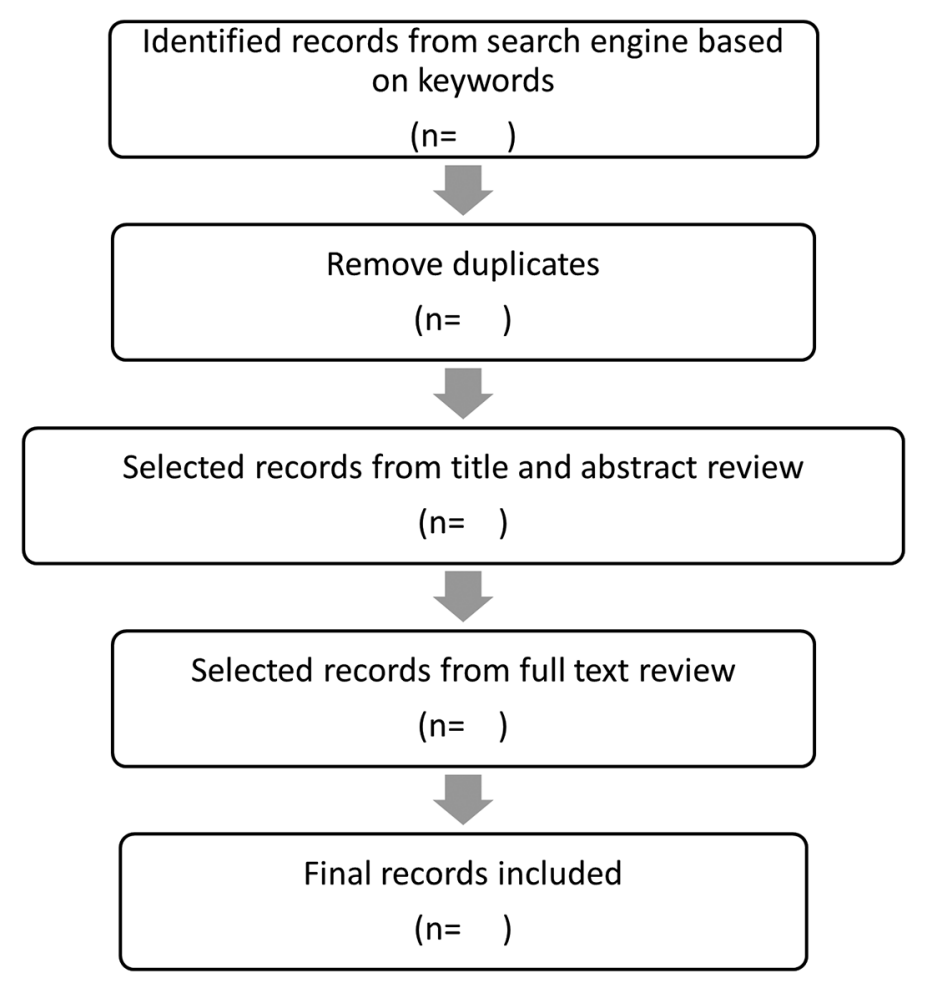

Figure 2. Steps of the article screening process.

To summarize the findings, a table will be developed mapping all the studies included in the scoping review. Studies will be categorized by different characteristics, including type of sources, study location, study design, as well as types of integration. EndNote 20 software will be used to store and managed the data obtained from the literature.

\section{Stage 5: Collating, summarizing, and reporting results}

After data extraction, a table will be developed to summarize and map the findings. Studies will be categorized based on several distinct characteristics. For example: study sample (patients, family or caregiver of patients, and healthcare providers), experience and perception of ideal concept for palliative care and good deaths, and level of healthcare service 
Table 3. Data extraction template.

\begin{tabular}{|l|l|}
\hline Data & Data description \\
\hline Article information & Author, year of publication, location of the study \\
\hline Type of sources & Peer-reviewed journal, grey literature, and international guideline \\
\hline Article type & $\begin{array}{l}\text { Quantitative, qualitative, mixed methods, guidelines, unpublished } \\
\text { reports }\end{array}$ \\
\hline Aim & Overall aim or objective of the study \\
\hline Population & Target population of the study \\
\hline Research methods & $\begin{array}{l}\text { Study design, sample size, methods of data collection, instruments used } \\
\text { in the study }\end{array}$ \\
\hline $\begin{array}{l}\text { Concept of palliative care and good } \\
\text { death of acute cases }\end{array}$ & $\begin{array}{l}\text { Definition and characteristics of palliative care and good death of acute } \\
\text { disease. }\end{array}$ \\
\hline Results & $\begin{array}{l}\text { Main findings of the study in the forms of quantitative results (\%, mean/ } \\
\text { median/standard deviation of some indicators, Odds Ratio, 95\% CI). For } \\
\text { qualitative studies we will include information on code, themes, and } \\
\text { important quotes for further analyses. }\end{array}$ \\
\hline Additional information & $\begin{array}{l}\text { Additional information of the study that relevant to the scoping review, } \\
\text { for example study limitation and recommendation. }\end{array}$ \\
\hline
\end{tabular}

where the palliative care is provided, or death occurred. Qualitative analyses by using thematic coding will be conducted to present a robust summary of the literature on experience and perception regarding palliative care and good death in acute diseases.

\section{Stage 6: Conducting consultation}

The results will be consulted to the experts and relevant stakeholders, which include patients, families, and caregivers. As this scoping review will be the basis for recommendation on palliative care and good death for people with acute diseases in Indonesia, relevant stakeholders will be identified from Indonesia. This step is important to ensure that the finding is validated, receive feedback and obtain additional insights into the findings. In the discussion, findings from the scoping review will be presented with feedback by relevant stakeholders provided.

\section{Ethics and dissemination}

We have obtained ethical permits for this review. The results of this review can be used as a reference for preparing a pilot study on integrated management of acute disease palliative care and good death management as well as policy briefs, presentations in conferences, peer-reviewed journals, and information on related websites.

\section{Study status}

This study is now at early stage of the step two of the scoping review process: identification of relevant studies. We are still searching for literature for relevant studies and abstracting data from the search engines. We estimated the scoping review to be completed by mid-2022.

\section{Data availability}

No data are associated with this article.

\section{References}

1. Reymond L, Parker G, Gilles $L$, et al.: Home-based palliative care. Aust. J. Gen. Pract. Nov. 2018; 47(11): 747-752. PubMed Abstract | Publisher Full Text

2. World Health Organization (WHO): Integrating palliative care and symptom relief into primary health care: a WHO guide for planners, implementers and managers. Geneva: World Health Organization; 2018
3. Ireland AW: Access to palliative care services during a terminal hospital episode reduces intervention rates and hospital costs: a database study of 19707 elderly patients dying in hospital, 2011-2015. Intern. Med. J. May 2017; 47(5): 549-556. PubMed Abstract | Publisher Full Text

4. Arrieira ICdO, Thofehrn MB, Porto AR, et al.: Spirituality in palliative care: Experiences of an interdisciplinary team. 
Rev. da Esc. Enferm. 2018; 52: 1-7.

Publisher Full Text

5. Gaertner J, et al.: Effect of specialist palliative care services on quality of life in adults with advanced incurable illness in hospital, hospice, or community settings: Systematic review and meta-analysis. BMJ. 2017; 357: j2925. Publisher Full Text

6. Sagha Zadeh R, Eshelman P, Setla J, et al.: Strategies to Improve Quality of Life at the End of Life: Interdisciplinary Team Perspectives. Am. J. Hosp. Palliat. Med. 2018; 35(3): 411-416. PubMed Abstract | Publisher Full Text | Free Full Text

7. Mercadante $S$, Gregoretti $C$, Cortegiani A: Palliative care in intensive care units: Why, where, what, who, when, how. BMC Anesthesiol. 2018; 18(1): 106-106.

PubMed Abstract | Publisher Full Text | Free Full Text

8. Diop MS, Rudolph JL, Zimmerman KM, et al.: Palliative Care Interventions for Patients with Heart Failure: A Systematic Review and Meta-Analysis. J. Palliat. Med. 2017; 20(1): 84-92. PubMed Abstract | Publisher Full Text | Free Full Text

9. Kopecký O, Rusinová K, Kouba M, et al.: The role of palliative medicine in acute conditions. Intern. Med. 2021; 65(schéma 1): 449-455.

Publisher Full Text

10. Luckett T, Phillips J, Agar M, et al.: Elements of effective palliative care models: A rapid review. BMC Health Serv. Res. 2014; 14: 1, 1-22. Publisher Full Text

11. Chamberlain S, et al.: Mortality Related to Acute Illness and Injury in Rural Uganda: Task Shifting to Improve Outcomes. PLoS One. 2015; 10(4): e0122559.

PubMed Abstract | Publisher Full Text | Free Full Text

12. Chowdhuri PD, Kundu K: Factors determining choice of complementary and alternative medicine in acute and chronic diseases. J. Complement. Integr. Med. 2020; 17(3): 1-10. PubMed Abstract | Publisher Full Text

13. Kellett J: Acute hospital medicine - A new sub-speciality or internal medicine re-born?. Eur. J. Intern. Med. 2011; 22(4): 334-338.

PubMed Abstract | Publisher Full Text

14. Meier EA, Gallegos JV, Montross-Thomas LP, et al.: Defining a Good Death (Successful Dying): Literature Review and a Call for Research and Public Dialogue. Am. J. Geriatr. Psychiatry. 2016 Apr.; 24(4): 261-271.

Publisher Full Text

15. Campbell SM: Well-Being and the Good Death. Ethical Theory Moral Pract. Aug. 2020; 23(3-4): 607-623. Publisher Full Text

16. Miyashita M, Morita T, Sato K, et al.: Good Death Inventory: A Measure for Evaluating Good Death from the Bereaved Family Member's Perspective. J. Pain Symptom Manage. 2008; 35(5): 486-498.

PubMed Abstract | Publisher Full Text

17. Powell T, Hulkower A: A Good Death. Hastings Cent. Rep. Jan. 2017; 47(1): 28-29. Publisher Full Text

18. Borgstrom $\mathrm{E}$ : What is a good death? A critical discourse policy analysis. BMJ Support. Palliat. Care. Jul. 2020; bmjspcare-2019002173.

Publisher Full Text

19. Arksey H, O'Malley L: Scoping studies: towards a methodological framework. Int. J. Soc. Resear. 2005; 8(1): 19-32. Publisher Full Text

20. The Joanna Briggs Institute: The Joanna Briggs Institute Reviewers' Manual 2015: Methodology for JBI scoping reviews. Joanne Briggs Inst. 2015 


\section{Open Peer Review}

\section{Current Peer Review Status:}

\section{Version 1}

Reviewer Report 14 December 2021

https://doi.org/10.5256/f1000research.78154.r101929

(C) 2021 Khalil H. This is an open access peer review report distributed under the terms of the Creative Commons Attribution License, which permits unrestricted use, distribution, and reproduction in any medium, provided the original work is properly cited.

\section{Hanan Khalil}

School of Psychology and Public Health, La Trobe University, Melbourne, Vic, Australia

Thank you for the opportunity to review this manuscript. I have the following comments for your consideration:

1. I am unclear to which methodology the authors are using, as they quoted both Arksey and Malley and JBI. Please specify.

2. Please specify the study types that you are including in the review.

3. The research questions are best addressed by a qualitative review rather than a scoping review.

4. The questions included are mainly qualitative in nature. Scoping reviews mainly address broader questions addressing; Participants, concept and context. The context is not mentioned in the review.

5. Please specify the rationale for conducting the scoping review as opposed to other types of review.

Is the rationale for, and objectives of, the study clearly described?

Yes

Is the study design appropriate for the research question?

Partly

Are sufficient details of the methods provided to allow replication by others? No

Are the datasets clearly presented in a useable and accessible format?

Not applicable 
Competing Interests: No competing interests were disclosed.

Reviewer Expertise: Health services research and palliative care

I confirm that I have read this submission and believe that I have an appropriate level of expertise to state that I do not consider it to be of an acceptable scientific standard, for reasons outlined above.

The benefits of publishing with F1000Research:

- Your article is published within days, with no editorial bias

- You can publish traditional articles, null/negative results, case reports, data notes and more

- The peer review process is transparent and collaborative

- Your article is indexed in PubMed after passing peer review

- Dedicated customer support at every stage

For pre-submission enquiries, contact research@f1000.com 